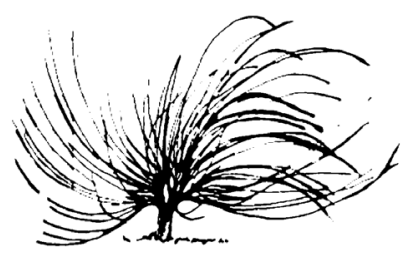

\title{
El uso de las TIC como recursos pedagógicos-metodológicos en el proceso de formación del estudiantado universitario del siglo XXI
}

\author{
Alejandro Fonseca Cascante ${ }^{1}$ \\ Ministerio de Educación Pública \\ Costa Rica \\ cfja27@gmail.com
}

\begin{abstract}
Resumen
Las tecnologías de la información y la comunicación (TIC) han llegado a convertirse en recursos de uso cotidiano para la población, por ello surge la necesidad de integrarlas en el ámbito educativo superior con el propósito de obtener el máximo provecho de ellas, pues estas ofrecen una serie de herramientas que resultan de suma utilidad en el desarrollo del proceso formativo. Los seres humanos nunca dejan de aprender, por lo tanto, las TIC se encuentran en constante evolución para adaptarse a las nuevas demandas y necesidades del mundo actual. Los centros educativos superiores, conscientes de esta realidad, consideran fundamental que los docentes se capaciten y recurran a la aplicación de estas tecnologías en sus clases con la finalidad de que la población estudiantil reconozca sus diferentes aportes y el apoyo que estas brindan en su desarrollo profesional, ante un indudable panorama cambiante en donde es necesario transitar de una sociedad de la información a una del conocimiento; de este modo, es posible evitar quedarse rezagado, buscando la manera de adaptarse a la era digital.
\end{abstract}

\section{(C) $\mathbb{Q} \Theta \Theta$}

http://dx.doi.org/10.15359/rep.esp-21.1

1 Profesor de secundaria en la modalidad diurna y nocturna en el Ministerio de Educación Pública Costa Rica. Licenciado en la Enseñanza de los Estudios Sociales y la Educación Cívica. Egresado de la Universidad Nacional de Costa Rica. 
Palabras claves: TIC, docentes, estudiantes, educación superior, currículo, pedagogía e innovación.

\begin{abstract}
Information and Communication Technologies (ICTs) have become resources for everyday use for the population; thus, the need to integrate them into the higher education field in order to obtain their maximum benefits possible emerges. Therefore, it cannot be denied that they offer a series of tools that can be used by students in the development of their training process. Human beings never stop learning; hence, ICTs are constantly evolving to adapt to the new demands or needs that individuals are presented with. Higher education centers, aware of this reality, consider it essential that teachers are trained and resort to the application of these in their classes, so that the student population recognizes their different contributions and the support they can provide in their professional development, before an undoubtedly changing landscape, where it is necessary to move from an information society to one of knowledge, so as not to lag behind and be able to adapt to the digital era that the global world is promoting.
\end{abstract}

Keywords: ICTs, teachers, students, higher education, curriculum, pedagogy and innovation.

\title{
Introducción
}

$\mathrm{E}$

1 ser humano, al desenvolverse en una sociedad en constante transformación, debido al fenómeno global que envuelve al mundo, demanda de una mayor preparación en los diferentes ámbitos de la vida cotidiana para poder dar respuesta o resolver las situaciones que se le presentan actualmente. El sistema educativo superior en Costa Rica no escapa de esta realidad, por tal razón surge la necesidad de reestructurar el currículo en pro de un proceso formativo que resulte eficiente ante las demandas y desafíos de la coyuntura actual, tal como lo afirma la autora Díaz (2008): 
es innegable el crecimiento en términos de cobertura de la educación virtual y de la incorporación de las TIC en diversos contextos educativos, presenciales y no presenciales, mostrando un aumento significativo en los próximos años, debido a que el avance tecnológico no se detiene dejando en evidencia que las posibilidades del empleo de las tic en la educación son mucho más amplias en relación con sus usos actuales. (p. 3)

Tomando en consideración lo anterior, resulta necesario formularse las siguientes interrogantes ante el proceso formativo del estudiantado del siglo XXI: ¿cómo las TIC han revolucionado la pedagogía en la última década?, ¿por qué los docentes del siglo XXI deben planear clases apoyándose en los distintos recursos tecnológicos?, ¿cómo pueden las TIC convertirse en metodologías que verdaderamente contribuyan a la formación del estudiantado universitario?, ¿cuál es el impacto actual de las TIC en el sistema educativo superior?, pues, como sostienen los autores Ramírez et al.:

los impactos de las TIC y de los procesos de globalización, son cada vez más significativos en las Instituciones de Educación Superior (IES) las cuales se han visto en la necesidad de plantear estrategias, mecanismos y procedimientos para adaptarse y responder a los retos de los contextos globales y emergentes, llamándole a esto "Internacionalización de la Educación Superior. (2015, p. 6)

El sistema educativo superior debe evolucionar, ya que solo de esta manera logrará romper con las metodologías tradicionalistas del proceso de enseñanza, reconociendo que existen otras maneras mediante las cuales los estudiantes puedan aprender, por lo tanto, el propósito de este trabajo es informar y reflexionar sobre cómo las TIC deben ser consideradas alternativas pedagógicas con la capacidad de contribuir en la construcción del conocimiento, promoviendo al mismo tiempo la formación de profesionales críticos, propositivos y participativos en un entorno altamente dinámico, porque sin duda alguna "la innovación es un proceso de destrucción creadora" (Díaz, 2008, p. 9), o sea, los actores involucrados acceden a la información y aportan desde sus propias creaciones. 
Asimismo, es importante que las personas dedicadas a la docencia reflexionen sobre su propia praxis para que logren entender la educación, no solo como un proceso de reproducción de saberes, sino también como un recurso que permite a cada individuo ampliar su conocimiento a partir de la interacción con los demás, potenciando sus distintas capacidades-habilidades con la implementación de la tecnología; esta última entendida como parte del escenario donde se desenvuelve cada persona. Mediante este entendimiento es posible potenciar su uso en la obtención de información y en la construcción de propuestas que puedan darse a conocer en otros lugares, a través de los distintos dispositivos tecnológicos con lo que se cuenta.

Ante este panorama, también es necesario que las instituciones de educación superior se transformen y adapten a los cambios para hacer frente a las nuevas demandas educativas de las sociedades del conocimiento (González, 2008, p. 4). Lo anterior, basado en el hecho de que el ámbito educativo es un espacio que no detiene su evolución, por lo cual, las personas deben ser conscientes de esta realidad, con el objetivo de no crear resistencias, sino más bien oportunidades, a partir de las cuales se pueda evidenciar el aporte de las tecnologías emergentes dentro de los distintos quehaceres que les corresponde llevar a cabo, siendo el ámbito educativo uno de los más favorecidos con el surgimiento y avance de las TIC. Esto en cuanto a que reta a los docentes universitarios a reinventarse para contribuir con el desarrollo de una práctica innovadora que le demuestre al estudiantado las diferentes experiencias de aprendizaje que se pueden tener con la integración de los recursos tecnológicos en el proceso de enseñanza.

\section{La integración de las TIC en el ámbito educativo superior}

La educación del siglo XXI requiere de una pedagogía reflexiva e innovadora, a partir de la cual los docentes sean conscientes de qué aspectos de su praxis deben cambiar con el objetivo de lograr, desde un enfoque epistemológico, la construcción de un conocimiento, no por repetición o transmisión de información, sino más bien producto de un intercambio de ideas en donde se reconoce al estudiantado como un sujeto autónomo que puede compartir sus saberes, ampliarlos y complementarlos con otros, permitiéndole adquirir un mayor enriquecimiento teórico, necesario para comprender las diversas temáticas que se pueden abordar desde los diferentes espacios en donde se desenvuelve, 
apostando de esta manera al desarrollo integral que se requiere con el principal propósito de atreverse a enfrentar el mundo y tomar decisiones por sí mismos.

Sin embargo, para poder llevar a cabo lo que se comenta en el párrafo anterior, es importante que los docentes promuevan el uso de las tecnologías de la información y comunicación (TIC), pues el estudiantado, además de obtener un repertorio abundante de información, puede canalizar y analizar la información, compartiéndola en diferentes espacios virtuales e interactuando con otros pares, permitiendo la construcción de un conocimiento interesante y enriquecedor entre todos los participantes, tal como lo afirman los autores Castro et al. (2007):

la incorporación de las TIC en el ámbito educativo es una de las actuales demandas de la sociedad del siglo XXI, ya que el conocimiento debe construirse tomando en consideración estructuras flexibles entre los cuales se destacan los entornos virtuales (TI$\mathrm{C}^{\prime}$ s), que se convierten en espacios que efectivamente incentivan el desarrollo de un aprendizaje, que bien direccionado promueve el desarrollo de un pensamiento crítico que permite la interpretación de la información en la generación de nuevo conocimiento, por parte de cada uno de los participantes del proceso de enseñanza y aprendizaje que se está llevando a cabo. (p. 4)

Las líneas anteriores dejan en evidencia que la coyuntura actual demanda de un sistema educativo que no puede estar desvinculado del uso de las TIC, porque la población estudiantil del presente siglo se encuentra muy identificada con ellas y las considera parte de su vida cotidiana; de esto surge la necesidad de aprovecharlas en la construcción del conocimiento del estudiantado, a partir de la aplicación de diferentes herramientas virtuales que promuevan la interacción, la creatividad, la difusión y la relación de información desde distintas percepciones o puntos vista, enriqueciéndose la dinámica pedagógica propuesta por el personal docente.

No obstante, para que lo anterior sea una realidad, las TIC deben ser consideradas estrategias que respondan a la formación de los individuos con competencias necesarias para la vida, el trabajo y el mundo, ante lo cual, sin duda alguna, se requiere de la potencialización de las habilidades comunicativas para promover la participación, la crítica y la 
reflexión en los sujetos participantes, quienes ven en estas herramientas recursos de gran apoyo en la realización de las diferentes asignaciones que deberán asumir durante su recorrido por la universidad. Así, en palabras de Ceballos et al. (2017):

TIC's se le debe considerar como herramientas fundamentales en muchos aspectos de la vida y en especial en la educación, ya que se han convertido en instrumentos didácticos que sabiéndolas utilizar mejoran el nivel de aprendizaje de los estudiantes, ya que permiten que se transformen de individuos considerados objetos de enseñanza a ser sujetos de aprendizaje. (p. 16)

Sin duda alguna, los recursos informáticos son de uso cotidiano para la población, es por ello que los docentes deben asumir este reto e integrar a estos dentro del proceso de enseñanza para lograr alcanzar resultados que sean favorables en el proceso formativo de los educandos. Se debe estar consciente de que la transformación del mundo demanda que las personas se involucren en este tipo de iniciativas y aprovechen al máximo los medios tecnológicos que surgen, para realizar las distintas labores que les corresponde y que les permite, al mismo tiempo, el desenvolvimiento como seres humanos en constante formación; por esta razón, resulta evidente la necesidad de estar dispuesto a querer involucrarse con las herramientas tecnológicas que se tienen o ponen a su disposición, en cuanto:

los educadores pueden mejorar los procesos de enseñanza-aprendizaje mediante el desarrollo de nuevas metodologías donde las tecnologías de la información y la comunicación (TIC) juegan un rol importante, ya que sirven como recursos de apoyo que permitirían promover el aprendizaje y generar nuevos espacios educativos, puesto que las TIC por sí solas no generan ningún tipo de aprendizaje; de ahí la importancia de hacer un uso pedagógico de ellas y conocer sus características principales con evidentes implicaciones educativas. (Rivero et al., 2016, p. 4)

En otras palabras, las TIC se ponen a disposición de los docentes para que estos potencien su utilización en el quehacer pedagógico, haciendo uso de las diferentes herramientas que las mismas proporcionan, 
con lo cual se evidencia la posibilidad de aprender mediante formas alternativas si se recurre a la aplicación de los recursos tecnológicos actuales. Además, es necesario inculcar la autonomía en el estudiantado universitario, ya que solo de esta manera las personas pueden adquirir consciencia de cuáles son sus verdaderas habilidades y destrezas para responder a las diversas dinámicas que se presenten en su día a día.

\section{La docencia universitaria: alianza entre la pedagogía y las TIC}

La docencia universitaria, actualmente, no puede aferrarse a una sola metodología de trabajo, pues estaría negándole de este modo a la población estudiantil la oportunidad de aprender de otras maneras; el mundo no detiene su evolución, por lo tanto, las exigencias actuales demandan de una propuesta curricular innovadora en donde los profesores pongan en acción didácticas que permitan atender los requerimientos actuales, en pro de un proceso formativo que promueva la interacción de los participantes; de esta forma se daría lugar a la construcción de un conocimiento a partir de la utilización de diferentes herramientas tecnológicas que se pueden emplear, obteniéndose resultados sumamente enriquecedores.

Por esta razón, el docente del siglo XXI debe ser praxeológico, pues solo así podrá repensar sus estrategias y llevar a cabo una autoevaluación de lo que se está haciendo en el contexto áulico y, así, reinventar su quehacer pedagógico, formulando como su principal objetivo la consolidación de un aprendizaje significativo. En relación con esta posición, es importante hacer referencia a lo que mencionan las autoras Fallas y Zúñiga (2010), quienes anotan que:

el desarrollo de las TIC ha venido a transformar sustancialmente el proceso educativo de las formas de aprender y lo que hay que aprender; las formas de representar, construir y comunicar el conocimiento; las formas de comunicación e interacción entre las personas y los medios para la integración y la actuación de los individuos dentro del tejido social, recalcando que las políticas e iniciativas adoptadas por los sistemas educativos de los diversos países en relación con la introducción y el uso de las tecnologías digitales en el ámbito educativo tienen que evidenciar las distintas visiones sobre sus formas de aprovechamiento y sobre los principales objetivos que se pueden alcanzar con la implementación y potencialización de las mismas. (p. 3) 
El ámbito educativo superior costarricense no puede ignorar esta realidad promovida por el fenómeno globalizador, sino que, por el contrario, la docencia universitaria debe incorporar y promover dentro de su práctica pedagógica el uso de las TIC como herramientas facilitadoras y promotoras de un conocimiento en constante ampliación. Ante esto, surge la necesidad de analizar y canalizar entre pares la información a la cual se posee acceso, para comprender realmente lo que sucede alrededor y no asumir todo lo que se difunde en los distintos medios tecnológicos. Sin duda alguna, los recursos tecnológicos se ponen a disposición de la población, quien posee la oportunidad de aprovecharla al máximo para obtener todos los beneficios que le sean posible.

Por otro lado, en los cursos universitarios se deben crear espacios reflexivos a partir de los cuales los docentes se percaten de la alianza que debe existir entre la pedagogía y las TIC, con el propósito de plantear estrategias metodológicas que sirvan para transformar el currículo educativo en aras de un aprendizaje significativo y atinente a las demandas del presente, es decir, que cale en el estudiantado y que le sirva en su desenvolvimiento. Para lograr este propósito "es necesario ir introduciendo cambios e innovaciones que capten la atención de las personas y contemplar el contexto en el que se interactúa" (Ramírez y Castilla, 2015, p. 17), para proponer metodologías que se apliquen a la realidad en la que se desenvuelve la población.

De igual manera, la propuesta anterior recalca entonces que el currículo del sistema educativo superior debe contemplar el recurso tecnológico como aquel medio que permite fortalecer la creatividad, la interacción entre los participantes, así como lograr desarrollar en los estudiantes la criticidad en cada información recibida o encontrada en la red, procurando la consolidación de un filtro personal en el estudiantado de todo aquello que les es dado como cierto, con el principal objetivo de dar paso al desarrollo de una consciencia propia y propositiva ante las diversas coyunturas a las que se enfrenta.

Asimismo, para generar un mayor impacto a nivel educativo, es fundamental contextualizar el uso de las TIC como instrumentos didácticos, porque solo así se logra que las mismas respondan de manera asertiva a las exigencias del entorno global en el que actualmente se vive, el cual evoluciona de manera progresiva, considerándose también elemental la disposición a querer renovarse, para responder atinadamente a las situaciones que se presentan. 
En síntesis, es necesario apostar a una pedagogía praxeológica, porque solo así se puede trascender más allá de lo que se hace en el aula. Resulta evidente que el hecho de ejecutar en las comunidades del país las distintas investigaciones, proyectos y programas que se llevan a cabo en el ámbito universitario resulta más enriquecedor para los estudiantes, pues se les permite interactuar, socializar, construir y concientizarse en relación con lo que sucede a su alrededor, incentivarlo a querer marcar la diferencia a partir del aporte que puede brindar y construir en él la necesidad de mejorar su entorno. En otras palabras, "promover la autonomía y la participación activa en el proceso educativo tiende a favorecer el potencial humano y la consolidación de una sociedad atinente a la realidad" (Vargas, 2013, p. 86).

Es indudable la importancia de incluir en la educación superior nuevas prácticas y modelos que sean pertinentes con las demandas de la sociedad actual, por ello, tanto la institución universitaria como el cuerpo docente son responsables de buscar una continua capacitación en el tema de las tecnologías emergentes, para así lograr una formación que responda a los constantes cambios que involucran el uso de la tecnología, en pro de agilizar los quehaceres que corresponde llevar a cabo. Además, es indispensable que las diferentes modalidades de la educación superior vayan incursionando en el uso de estrategias $b$-learning, puesto que estas nuevas tecnologías son clave para potenciar el proceso de aprendizaje mediante el diseño de materiales didácticos de calidad en cuanto:

la expansión de las TIC's en la era universitaria claramente ha marcado una diferenciación entre el estudio tradicional, ya que es posible realizar las diferentes labores en un menor tiempo y apoyarse en estructuras más dinámicas que faciliten la explicación que se está compartiendo con los demás miembros. (Fernández, 2014, p. 27)

De este modo, innovar se convierte en una necesidad-prioridad dentro del ámbito educativo superior, pues, a partir de este momento, los docentes se atreven a romper esquemas pedagógicos tradicionalistas y aprovechan los distintos medios tecnológicos que tienen a su alrededor en la formación de los futuros profesionales, sin importar la carrera en la que se encuentren las personas para ampliar su conocimiento y a 
la vez compartir pensamientos-criterios en los espacios virtuales que las herramientas tecnológicas ponen a su disposición, como por ejemplo el uso de plataforma digitales en donde se pueden realizar actividades como foros, wikis, videoconferencias, paisajes de aprendizaje e intervenciones colaborativas.

De igual manera, en las universidades se manejan diversos tipos de procesos cada vez más innovadores en la forma en que se utilizan las TIC, siendo de uso presencial, semipresencial y virtual algunas de las modalidades que se ofrecen como alternativas educativas. Ante esta posición, es importante hacer hincapié en que los docentes son los encargados de generar el principal cambio, para, de esta manera, crear espacios tecnológicos y virtuales en los que los y las jóvenes se familiaricen con su uso, incentivando a una ruptura de las barreras u obstáculos que puedan existir con respecto al uso de las herramientas tecnológicas, sin embargo, esto solo puede ser posible cuando:

La implementación de las TIC vaya de la mano con el currículo del docente para garantizar que se dé la educación acorde a lo que el programa requiera, involucrando la tecnología que facilite la educación como principal objetivo de esta. La integración de la información debe hacerse de la manera correcta, porque de nada vale toda la información que se puede conseguir a través de ellas si no se usa correctamente. (Díaz, 2008, p. 9)

Para lograr que las TIC tengan un impacto significativo en el ámbito educativo superior es necesario que los docentes se comprometan con una permanente formación y participen en capacitaciones a través de las cuales puedan informarse sobre las herramientas que tienen a su disposición y aprendan a utilizarlas con el objetivo de ejecutarlas efectivamente en el desarrollo de sus clases. Además, es necesario que el currículo contemple esta realidad y la considere un eje transversal, para que los participantes del proceso de enseñanza y aprendizaje las implementen en las diferentes asignaciones formativas que deberán realizar en cada uno de los cursos que matriculen durante su estancia en la universidad, para lo cual se requiere que:

los docentes sigan capacitándose conforme los cambios que el sistema vaya implementando, informándose y adaptándose al 
cambio tecnológico. La tecnología se ha vuelto clave esencial para el aprendizaje, los docentes tienen la ventaja de combinar sus técnicas en las aulas, para que el aprendizaje sea más hábil y efectivo, los docentes buscan cambiar sus métodos, y esto implica buscar en la tecnología complementos para que el aprendizaje sea más llevadero e implique menos esfuerzo en las aulas, los docentes pueden unificar ambos estilos, llevando a cabo una educación exitosa y que genera un grado de satisfacción significativo en el estudiantado que se encuentra empleándola. (García et al., 2015, p. 91)

Así, es posible afirmar que las TIC tienen como propósito convertirse en herramientas trascendentales para los docentes, facilitando la manera en la que se van dando las situaciones cada día, permitiéndole a la población estudiantil experimentar a través de otras técnicas metodológicas y bajo otro nuevo enfoque-paradigma educativo, es decir, "la universidad y particularmente los profesores universitarios deben contribuir, con una práctica educativa innovadora, para coadyuvar a transitar de la sociedad de la información a las sociedades del conocimiento" (González, 2008, p. 2).

Los docentes universitarios, conscientes de la sociedad evolutiva en la que se desenvuelven, no deben perder de vista la permanente formación que demanda su profesión, para evitar estructurar y dirigir la educación hacia una sola dirección, para lo cual, detenerse y realizar una introspección consciente de su labor resulta fundamental para evitar aferrarse a una praxis que no genere ningún reto cognitivo. Ante este panorama, Abarca (2015) anota:

con la implementación de las TIC'S se busca aplicar métodos para cambiar rotundamente la forma en la que se ven las clases curriculares, resaltando la necesidad de que se aplique la tecnología para llevar a casa la educación, ser más abiertos y tener una comunicación amplia, porque se implementan métodos de las TIC'S novedosos que le permiten al docente convertirse en el puente para que el estudiante aborde la tecnología, siendo guía y supervisor de que el material de enseñanza llegue tal cual los propósitos del programa de estudio así lo requieran. (p. 338) 
El proceso de innovación, cabe agregar, involucra una serie de mecanismos que van más allá de colocar una computadora o un proyector de video en clase; en este sentido, los docentes deben ser conscientes de la gran variedad de herramientas que los recursos tecnológicos ofrecen para promover en el estudiantado su utilización, pues a partir de su integración es posible resolver una gran variedad de problemas e interrogantes que surgen en el proceso de enseñanza, diversos proyectos, tareas y trabajos, hecho que genera un mayor impacto en el proceso de aprendizaje, siendo el docente el facilitador-mediador, cuyo propósito principal es alcanzar resultados gratificantes.

De igual manera, el reto académico del docente actualmente consiste en la creación de paisajes de aprendizaje, tomando en consideración las necesidades, la motivación y el contenido vinculado al desarrollo educativo de la población estudiantil, sin embargo, esto solo será posible a partir de una transformación curricular que genere el impacto que se requiere, para lo cual el docente y los estudiantes deben estar también dispuestos a querer involucrar los recursos tecnológicos en el proceso educativo y eliminar las actitudes de resistencia que son las que impiden la visualización de la transformación pedagógica a la que es necesario apostar actualmente. En el mismo orden de ideas se resalta el hecho de que:

Las TIC han transformado el entorno de aprendizaje actual, pasando de uno tradicional centrado en el docente a uno centrado en el estudiantado, ya que el primero ha dejado de ser la principal fuente de información y el principal emisor de conocimiento, para convertirse en un guía o mediador del proceso de enseñanza y aprendizaje, mientras que el alumno ha pasado de ser un receptor pasivo de información a convertirse en un sujeto que participa activamente en la consolidación de su propio aprendizaje. (Castro et al., 2007, p. 223)

Resulta evidente que las TIC están revolucionando el sistema educativo al ofrecer nuevas herramientas de aprendizaje y enseñanza que permiten soluciones útiles para la educación y la formación permanente de las personas, ampliando sus conocimientos para comprender lo que sucede a su alrededor. Las TIC surgen como apoyo a los distintos quehaceres de la población, por lo tanto, deben ser consideradas un 
aliado importante en su efectivo, íntegro y placentero desenvolvimiento en la sociedad, en cuanto "la tecnología es una parte indesligable de la sociedad, de modo que los dispositivos y sistemas tecnológicos se moldean socialmente a la vez que moldean a la sociedad" (Loveless y Williamson, 2017, p. 6).

El uso de tecnologías viene a complementar o, incluso, a remplazar en muchas ocasiones las clases totalmente magistrales que generan, en gran parte de la población estudiantil, tedio y descontextualización. En cuanto al acceso a la tecnología, se puede afirmar que, aunque esta no es accesible a la totalidad de la población, sí presenta una mayor apertura de acceso en comparación con épocas anteriores y una mayor facilidad de implementación.

Asimismo, los y las docentes que implementan las TIC en sus clases brindan a sus estudiantes un mayor espectro de oportunidades creativas que benefician la actitud con la que estos se relacionan con el proceso de aprendizaje al tener disponibles una gran cantidad de herramientas y sistemas de innovación que resultan aptos para la experimentación, lo cual favorece a una mejor disposición por parte de la población estudiantil. Esto debido a que:

la sociedad actual y cada vez más la educación se encuentran permeadas por un estilo de pensamiento cibernético, en donde la concepción del aprendizaje, la pedagogía y el currículo se han ido modificando según las demandas del mundo globalizado y el vínculo cibernético, como la virtualidad, las redes y la conexión las cuales configuran el futuro de la educación. (Loveless y Williamson, 2017, p. 20)

La interconexión en la cual se encuentran inmersos los seres humanos brinda la oportunidad de interactuar a través de los distintos medios, pudiéndose visualizar los diferentes espacios en los cuales es posible generar conocimiento y exponer proyectos que permitan transformar el escenario para hacerle frente a los nuevos retos y desafíos que se presentan en la sociedad, esto desde una correcta potencialización de las capacidades que permite plantear soluciones y presentarlas al público mediante los recursos tecnológicos que se tienen a disposición, en donde la distancia no es un obstáculo para relacionarse con otras personas. 
De igual manera, aunque "la aparición de las Tecnologías de la Información y la Comunicación (TIC) ha incidido en las funciones del sistema de educación y permitido la innovación en la ampliación-construcción de nuevos saberes" (García et al., 2017, p. 5), el docente es quien debe establecer su correcta integración en el proceso educativo con la finalidad de que el empleo de estas esté enfocado en investigar y construir trabajos interesantes y creativos, a partir de los cuales demuestren el abordaje de las temáticas que les corresponde comprender como parte de su proceso formativo; en otras palabras, el acceso a los recursos digitales tiene que ser amigable, no ser un arma que interrumpa los procesos de aprendizaje; por lo tanto, el docente debe buscar la manera de equilibrar en la forma en la que se distribuye la tecnología, para que esta no acapare la forma en la que se debe aprovechar y utilizar como recurso pedagógico, ya que en esencia "las nuevas tecnologías están diseñando nuevos espacios de enseñanza, nuevas normas, nuevos modelos que precisan de nuevos profesores que deberán ir ocupando este nuevo espacio" (González, 2008, p. 4).

El personal docente universitario actual, sin duda alguna, debería construir una identidad pedagógica que se encuentre vinculada con las nuevas tecnologías, pues la era digital en la que están inmersos demanda de un proceso formativo en donde el sujeto sea activo, autónomo y autorresponsable, con el propósito de cuestionar y proponer ideas en un mundo saturado de información, donde el análisis, el diálogo y el trabajo en equipo resultan ser cualidades indispensables en el proceso formativo.

Si bien en la actualidad debe enfrentarse a entornos cada vez más complejos y dinámicos, esto no debe ser motivo de frustración, por el contrario, comprometerse con la actualización permite convertir las nuevas tecnologías en recursos valiosos a través de los cuales es posible apoyar las prácticas pedagógicas y romper así con el esquema tradicional de escolarización. La construcción del conocimiento debe ser el principal propósito del sistema educativo, es por ello que, sin importar la disciplina, todo sujeto docente debe potenciar la consciencia y las habilidades para que el estudiantado aprenda a partir de lo que ya sabe, permitiendo que el proceso de enseñanza y aprendizaje se disfrute más y logre calar en cada uno de las personas involucradas, es decir, que tenga un mayor significado al dejar de lado lo mecánico y empezar a potenciar la capacidad de racionamiento y creación con la integración de las TIC en la educación. 
Asimismo, considerando lo mencionado en las páginas anteriores, se exponen en seguida algunos principios educativos y rasgos deseables en un nuevo paradigma de diseño educativo acorde a la sociedad de la información. Estos aspectos no corresponden únicamente a los entornos virtuales e híbridos; sino que también abarcan los cambios deseables en la educación presencial con la intención de dinamizarla y contribuir en la formación de los estudiantes del siglo XXI:

- Las herramientas tecnológicas tienen que convertirse en alternativas pedagógicas que permitan la construcción de conocimiento y promuevan el trabajo colaborativo entre las personas participantes de los cursos universitarios.

- Las TIC brindan espacios que se convierten en comunidades de aprendizaje porque les permite a los estudiantes interactuar y construir propuestas sumamente enriquecedoras en cuanto se pueden compartir para incentivar a otros a transformar el escenario al que pertenecen. Asimismo, las mismas también pueden ser utilizadas por los docentes como redes colaborativas en pro del desarrollo y la actualización académica.

- Es necesario desarrollar la alfabetización digital o tecnológica en las personas estudiantes y docentes; de igual forma, es debido vincularla a estrategias de pensamiento de alto nivel para que las personas puedan llevar a cabo un análisis, una búsqueda, un cuestionamiento, un descubrimiento y un planteamiento de propuestas que refleje la apropiación de su aprendizaje.

- Las tecnologías emergentes, sin lugar a dudas, pueden ofrecer clases presenciales que se caractericen por capturar la atención del estudiantado al ser más interactivas, pues, si la mediación resulta adecuada, el estudiantado tendrá un rol activo-participativo dentro de la clase, lo cual causará motivación para involucrarse cada vez más en la dinámica enseñanza-aprendizaje.

- Las estrategias o herramientas a utilizar deben de estar adaptadas a las necesidades del estudiantado de cada disciplina, recurriendo a las capacidades digitales del docente, ya que estas marcan las pautas para llevar a cabo un proceso de enseñanza y aprendizaje transformador.

- Los docentes universitarios deben potenciar las competencias tecnológicas en sus estudiantes, pues estas son indispensables en la 
formación de toda persona en pleno siglo XXI, la cual actualmente se enfrenta a una era digital que no detiene su evolución.

- Es necesario ofrecer la oportunidad de que los estudiantes desarrollen habilidades digitales, blandas y técnicas, ya que, de esta manera, se logra quesean competentes en su área; esto significa estar anuente a involucrarse con el recurso tecnológico.

- Efectivamente, los docentes deben convertirse en facilitadores del aprendizaje y no entes unilaterales en cuanto al ofrecimiento de la información a la persona estudiante. ¿Cuál podría ser una posible solución? fácil, capacitación docente en temas de neurociencia, inteligencias múltiples y metodologías activas a partir de la implementación de las TIC en el quehacer pedagógico.

- Las herramientas tecnológicas dan paso a la construcción de conocimiento de manera colectiva, pues, al utilizar aplicaciones o servidores en línea, se logra que varias personas intervengan en un mismo documento o producción.

- $\quad$ No se puede ignorar que la sociedad se transforma y las exigencias del estudiantado del siglo XXI son otras, por lo tanto, es necesario salir del estado de confort para apostar por un desarrollo de un proceso educativo continuo que pueda ser más enriquecedor, experimentando nuevas metodologías en la labor formativa que deriven en un compromiso innovador.

- Dejar claro que se deben aprovechar las diversas aplicaciones que ofrecen las nuevas tecnologías emergentes para apoyar el quehacer pedagógico, apostando de esta manera a un proceso educativo innovador en donde al estudiante se le insta a incorporar los recursos tecnológicos con los que cuenta para el desarrollo de su aprendizaje.

- Es necesario potenciar las diferentes competencias digitales en los estudiantes, para que comprendan, construyan, propongan y tomen decisiones por sí mismos; por esta razón, el escenario educativo no debe considerar las TIC como amenaza, sino como una oportunidad de crecimiento personal, integral y necesario para el pleno desenvolvimiento de las personas en un mundo altamente convulso y competitivo.

Sin duda alguna, resulta evidente la existencia de un gran reto para la población docente, principalmente para quienes no se han apropiado aún del uso de las tecnologías digitales en los procesos 
educativos. Como señala García (2016), los docentes "deben diseñar estrategias didácticas y ambientes de aprendizaje mediados por tecnologías de la información y la comunicación (TIC) para contribuir a que sus estudiantes -nativos digitales- desarrollen las competencias requeridas por la sociedad del siglo XXI" (p. 116). Por tanto, se debe no solo comprender y apropiarse del uso de la tecnología, sino también lograr transmitir en el proceso de enseñanza y aprendizaje, la funcionalidad de esta al estudiantado. Los espacios universitarios no deberían desligarse actualmente de los recursos tecnológicos que se encuentran su disposición en la construcción de un conocimiento mediante la interacción y el incentivo a la innovación.

\section{Conclusiones}

Reconocer el aporte de las tecnologías de la información y comunicación en el ámbito educativo es una de los principales objetivos del presente trabajo; esto debido a que resulta necesario tomar en consideración el gran impacto que tiene la tecnología en la actualidad en cuanto permite el acceso a diversas herramientas con la capacidad de dar respuesta a múltiples situaciones cotidianas y ser, además, recurso realmente útil para el aprendizaje y el desarrollo de competencias, hecho mediante el cual es posible que el proceso de aprendizaje trascienda más allá de las aulas.

De igual manera, no es posible negar que la tecnología ha venido a revolucionar el estilo de vida de los seres humanos y el sistema educativo superior no escapa de esta realidad; evidencia de ello es el proceso de virtualización al cual se apuesta en este momento, el cual demanda que los profesionales en docencia asuman con compromiso dicha coyuntura, capacitándose e involucrándose con el uso de las diferentes herramientas tecnológicas que potencian el aprendizaje, tal como afirma Abarca (2015):

la integración de las TIC'S han cambiado la forma de hacer docencia, porque brindan un acceso fácil, rápido y seguro a la información y al procesamiento de datos, lo cual ha significado una revolución en la educación, pues en el pasado para que un estudiante pudiera acceder a información fidedigna debía desplazarse a una biblioteca. (p. 338) 
Las personas, por lo tanto, no deberían resistirse a la modernización que promueve la innovación tecnológica, por el contrario, deberían incorporarla en su vida cotidiana y utilizarla para llevar a cabo los diferentes quehaceres de una manera más ágil y efectiva. En pocas palabras, las tecnologías de la información y la comunicación (TIC) pueden complementar, enriquecer y transformar la educación, convirtiéndose en herramientas pedagógicas de gran utilidad en la consolidación y reconstrucción del conocimiento.

Para poder llevar a cabo este propósito es importante que los y las docentes promuevan el uso de las tecnologías de la información y comunicación (TIC) con la finalidad de que el estudiantado, además de obtener un repertorio abundante de información, pueda canalizar y analizar los datos brindados en el proceso de enseñanza, compartiéndola en diferentes espacios virtuales e interactuando con otros pares, construyendo de esta manera un conocimiento interesante y enriquecedor para todos los participantes del proceso educativo. De igual manera, es importante resaltar que los entornos virtuales que brindan las TIC se deben considerar estructuras flexibles de enseñanza, cuya principal finalidad debe ser convertirse en recursos de apoyo ante la diversidad de ritmos aprendizaje que confluyen en un mismo espacio.

Es importante reconocer que tanto la población docente como la población estudiantil deben apostar a la innovación y atreverse a asumir nuevos retos pedagógicos que les permitan crecer como profesionales; ante esto, el empoderamiento y la motivación son dos elementos que deben estar presentes en el ámbito educativo:

quienes hemos permanecido en contacto con niños, adolescentes y jóvenes sabemos de lo desalentador que resulta una escuela basada en la anomia, la falta de contenidos nuevos y actualizados, con pocas actividades que desafíen la inteligencia y el razonamiento, que promuevan nuevas formas de organización social, la solidaridad, la conciencia y el compromiso entre otras cosas. (Sanjurjo y Rodríguez, 2005, p. 14)

Asimismo, el currículo debe ser una propuesta formativa que involucre la participación de los diferentes sectores, con el objetivo de enriquecerlo y que, al mismo tiempo, este responda a las diferentes necesidades del grupo al que se encuentra dirigido con el propósito de que 
estos puedan empoderarse e involucrarse en las distintas situaciones que se presenten, porque el mundo cambiante en el que se encuentran inmersos demanda que se atrevan a promover cambios, a partir de los cuales se pueda hacer frente a las nuevas exigencias que se plantean, esto para evitar quedarse rezagado en el panorama de innovación promovido actualmente por las TIC.

Asimismo, la praxis no debe contar con una determinada estructura que la mantenga rígida, por el contrario, esta debe estar dispuesta a experimentar otras metodologías y estrategias didácticas con la implementación de las TIC que permita evidenciar la existencia de otras formas de aprendizaje. Ante este panorama, la persona docente debe capacitarse en la utilización y en la enseñanza de las herramientas tecnológicas a favor de promover un intercambio eficiente con la población estudiantil, para así transmitir de forma adecuada las habilidades necesarias para el uso de TIC en el proceso de aprendizaje.

En conclusión, las prácticas pedagógicas, con respecto a lo planteado anteriormente, no deben mantenerse estáticas sino que deberían ser reflexionadas a pesar de nuestra cotidianidad en el aula, para atreverse a romper estructuras o paradigmas tradicionales que le impide al estudiantado ver más allá de lo que algunos quieren difundir, además de brindarle la posibilidad de compartir información con otros y otras colegas, adquirir métodos, nuevos procedimientos y estrategias tecnológicas que enriquecerán su aprendizaje.

Relacionado a esta necesidad, diversos autores y autoras, son enfáticos en la necesidad de repensar y reflexionar sobre las maneras en las que se ha pensado y se sigue aplicando la educación, las tecnologías y la creatividad; con el fin de poder aportar una mirada crítica a las posibles políticas, discursos y miradas de la educación, para así incidir en las maneras de conocer y actuar en la actual era digital.

Los docentes universitarios deben promover la construcción de redes de colaboración cibernéticas, a partir de las cuales puedan compartir estrategias pedagógicas que involucran la implementación de herramientas proporcionadas por las TIC con el propósito de abordar las nuevas exigencias y las nuevas necesidades que se presentan en el ámbito educativo. 


\section{Referencias}

Abarca, Y. (2015). El uso de las TIC en la educación universitaria: Motivación que incide en su uso y frecuencia. Revista de Lenguas Modernas, 22, 335-350.

Castro, S., Guzmán, B. y Casado, D. (2007). Las Tic en los procesos de enseñanza y aprendizaje. Red de Revistas Cientificas de América Latina, el Caribe, España y Portugal, 23(13), 213-235.

Ceballos H., Ospina L. y Restrepo J. (2017). Integración de las TIC en el proceso de enseñanza y aprendizaje. Universidad Pontificia Bolivariana.

Díaz, F. (2008). Educación y nuevas tecnologías de la información: ¿Hacia un paradigma educativo innovador? Revista Electrónica Sinéctica, 30, 1-15.

Fallas, I. y Zúñiga, M. (2010). Las tecnologías digitales de la información y la comunicación en la educación costarricense. Consejo Nacional de Rectores.

Fernández, D. (2014). El uso de las TICs y los estudiantes universitarios. Congreso Iberoamericano de Ciencia, Tecnología, Innovación y Educación. Universidad de Buenos Aires, Argentina.

García, C. L. (2016). Enseñar con TIC. Nuevas y renovadas metodologías para la enseñanza superior. https://www.cinep.ipc.pt/images/ensinar\%20con\%20tic.pdf

García, M., Reyes, J. y Godínez, G. (2017). Las Tic en la educación superior, innovaciones y retos. Revista Iberoamericana de Ciencias Sociales y Humanisticas, 12(6), 1-18.

García, C., Piña, J., Ancona, M. y Navarrete, M. (2015). las tecnologías de la información como un factor de mejora en el proceso de enseñanza-aprendizaje en la educación a distancia en una universidad. Revista Internacional de Tecnología, Conocimiento y Sociedad, 4(1), 85-95.

González, J. (2008). TIC y la transformación de la práctica educativa en el contexto de las sociedades del conocimiento. Revistas Científicas de América Latina, el Caribe, España y Portugal, l(5), 1-8. https://www.redalyc.org/pdf/780/78011201003.pdf

Loveless, A. y Williamson, B. (2017). Nuevas identidades de aprendizaje en la era digital. Creatividad, Educación, Tecnología y Sociedad. Ediciones Narcea. 
Ramírez, A., y Casillas, M.A. (2015). Háblame de TIC: Internet en Educación Superior. Editorial Brujas.

Ramírez, A., Morales, A. y Olguín, P. (2015). Marcos de referencia de saberes digitales. Revista de Educación Mediática y TIC, 4(2), 122136. https://helvia.uco.es/xmlui/bitstream/handle/10396/13514/ Edmetic_vol_4_n_2_8.pdf? sequence $=1 \&$ is Allowed $=y$

Rivero, C., Chávez, A., Vásquez, A. y Blumen, S. (2016). Las TIC en la formación universitaria. Logros y desafíos para la formación en psicología y educación. Revista De Psicología, 34(1), 185-199. https://doi.org/10.18800/psico.201601.007

Sanjurjo, L. y Rodríguez, X. (2005). Volver a pensar la clase. las formas básicas de enseñar (1ed). Homo Sapiens Ediciones.

Vargas, J. (2013). La pedagogía praxeológica: Hacia una plena realización del potencial humano. Corporación Universitaria Minuto de Dios. http://cnbmtucuman.edu.ar/cnbm_files/biblioteca_doc/Una Pedagogia_Praxeologica-Carlos_German_Juliao_Vargas.pdf 
